Jia-Le Li, Zhang-Fan Chen and Shui-Sheng Chen*

\title{
Crystal structure of catena-poly[diaqua-bis $\left(\mu_{2}-5-\right.$ (3-(1H-imidazol-5-yl)phenyl)tetrazol-2-ido- $\mathrm{K}^{2} \mathrm{~N}: \mathrm{N}^{\prime}$ ) cobalt(II)], $\mathrm{C}_{20} \mathrm{H}_{18} \mathrm{CoN}_{12} \mathrm{O}_{2}$
}

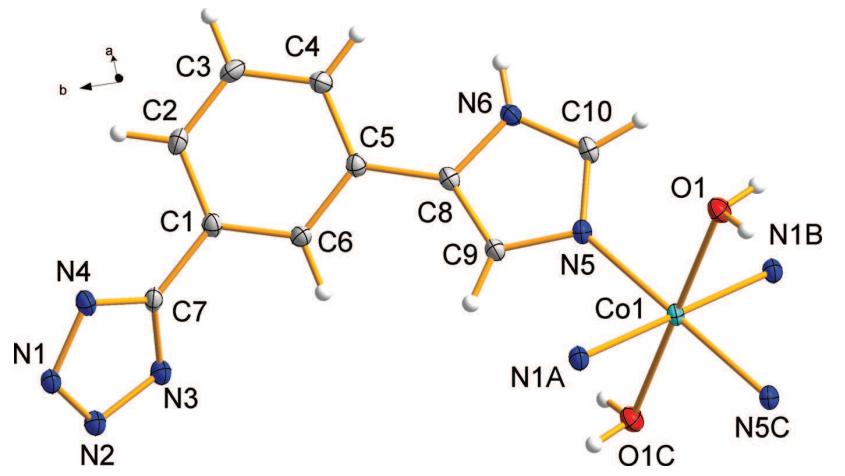

https://doi.org/10.1515/ncrs-2018-0148

Received June 25, 2018; accepted August 14, 2018; available

online September 5, 2018

\begin{abstract}
$\mathrm{C}_{20} \mathrm{H}_{18} \mathrm{CoN}_{12} \mathrm{O}_{2}$, monoclinic, $C 2 / c$ (no. 15), $a=17.6924(15) \AA$, $b=11.3253(10) \AA, \quad c=12.8536(11) \AA, \quad \beta=128.029(10)^{\circ}$ $V=2028.7(3) \AA^{3}, Z=4, R_{\mathrm{gt}}(F)=0.0258, w R_{\text {ref }}\left(F^{2}\right)=0.0890$, $T=296(2) \mathrm{K}$.
\end{abstract}

CCDC no.: 1862080

A part of the polymeric title crystal structure is shown in the figure $(\mathrm{a}=0.5-x, 1.5-y,-z ; \mathrm{b}=x, y-1, z ; \mathrm{c}=0.5-x$, $0.5-y,-z)$. Tables 1 and 2 contain details on crystal structure and measurement conditions and a list of the atoms including atomic coordinates and displacement parameters.

\section{Source of materials}

All reagents and solvents were used as obtained without further purification. A mixture containing Cobalt(II) chloride hexahydrate (0.023 g, $0.1 \mathrm{mmol}), 1-(1 H$-imidazol-4-yl)-3-(4Htetrazol-5-yl)benzene (HL) (0.021 mg, $0.1 \mathrm{mmol}), \mathrm{DMF}(1 \mathrm{~mL})$, $10 \mathrm{~mL} \mathrm{H}_{2} \mathrm{O}$ was sealed in a $16 \mathrm{~mL}$ Teflon-lined stainless steel

*Corresponding author: Shui-Sheng Chen, College of Chemistry and Chemical Engineering, Fuyang Normal University, Fuyang, Anhui 236041, China, e-mail: sscfync@163.com

Jia-Le Li: College of Chemistry and Chemical Engineering, Fuyang Normal University, Fuyang, Anhui 236041, China Fuyang, Anhui 236041, China
Zhang-Fan Chen: Department of Chemistry, Fuyang Normal College,

Table 1: Data collection and handling.

\begin{tabular}{ll}
\hline Crystal: & Purple block \\
Size: & $0.24 \times 0.20 \times 0.16 \mathrm{~mm}$ \\
Wavelength: & Mo $K \alpha$ radiation $(0.71073 \AA)$ \\
$\mu:$ & $0.90 \mathrm{~mm}^{-1}$ \\
Diffractometer, scan mode: & Bruker Apex, $\varphi$ and $\omega$ \\
$\theta_{\text {max }}$, completeness: & $25.0^{\circ}, 99 \%$ \\
$N(h k l)_{\text {measured }}, N(h k l)_{\text {unique }}, R_{\text {int }}:$ & $5322,1779,0.022$ \\
Criterion for $I_{\text {obs }}, N(h k)_{\text {gt }}:$ & $I_{\text {obs }}>2 \sigma\left(I_{\text {obs }}\right), 1608$ \\
$N(\text { param })_{\text {refined }}:$ & 164 \\
Programs: & Bruker $[1]$, SHELX $[2,3]$ \\
\hline
\end{tabular}

Table 2: Fractional atomic coordinates and isotropic or equivalent isotropic displacement parameters $\left(\AA^{2}\right)$.

\begin{tabular}{lrrrr}
\hline Atom & $\boldsymbol{x}$ & $\boldsymbol{y}$ & $\boldsymbol{z}$ & $\boldsymbol{U}_{\text {iso }}{ }^{*} \boldsymbol{U}_{\text {eq }}$ \\
\hline C01 & 0.250000 & 0.250000 & 0.000000 & $0.01885(16)$ \\
C1 & $0.44090(13)$ & $0.85089(17)$ & $0.04833(18)$ & $0.0213(4)$ \\
C2 & $0.53791(14)$ & $0.87934(17)$ & $0.13885(19)$ & $0.0277(4)$ \\
H2 & 0.557177 & 0.957950 & 0.154012 & $0.033^{*}$ \\
C3 & $0.60504(13)$ & $0.7913(2)$ & $0.2056(2)$ & $0.0319(5)$ \\
H3 & 0.669826 & 0.810586 & 0.263930 & $0.038^{*}$ \\
C4 & $0.57729(13)$ & $0.67413(18)$ & $0.18710(19)$ & $0.0267(4)$ \\
H4 & 0.623348 & 0.615332 & 0.233771 & $0.032^{*}$ \\
C5 & $0.48073(13)$ & $0.64403(16)$ & $0.09887(17)$ & $0.0206(4)$ \\
C6 & $0.41337(13)$ & $0.73363(16)$ & $0.0275(2)$ & $0.0222(4)$ \\
H6 & 0.348942 & 0.714215 & -0.035058 & $0.027^{*}$ \\
C7 & $0.36969(13)$ & $0.94588(16)$ & $-0.02047(18)$ & $0.0221(4)$ \\
C8 & $0.44866(12)$ & $0.52176(16)$ & $0.08262(17)$ & $0.0209(4)$ \\
C9 & $0.36021(13)$ & $0.47629(17)$ & $0.02707(19)$ & $0.0239(4)$ \\
H9 & 0.304502 & 0.520933 & -0.013874 & $0.029^{*}$ \\
C10 & $0.45505(13)$ & $0.32806(16)$ & $0.10178(18)$ & $0.0225(4)$ \\
H10 & 0.479076 & 0.251459 & 0.123003 & $0.027^{*}$ \\
N1 & $0.29323(11)$ & $1.10390(13)$ & $-0.05730(15)$ & $0.0235(4)$ \\
N2 & $0.24868(11)$ & $1.04857(14)$ & $-0.17092(16)$ & $0.0258(4)$ \\
N3 & $0.29627(11)$ & $0.94745(13)$ & $-0.15023(16)$ & $0.0260(4)$ \\
N4 & $0.37002(11)$ & $1.04133(14)$ & $0.03979(16)$ & $0.0252(4)$ \\
N5 & $0.36416(11)$ & $0.35517(14)$ & $0.03956(15)$ & $0.0224(4)$ \\
N6 & $0.50861(11)$ & $0.42456(13)$ & $0.13072(15)$ & $0.0220(4)$ \\
H6A & 0.569552 & 0.425791 & 0.171959 & $0.026^{*}$ \\
01 & $0.34393(10)$ & $0.17563(13)$ & $0.19713(13)$ & $0.0275(3)$ \\
H1 & 0.311541 & 0.145628 & 0.215907 & $0.041^{*}$ \\
H1A & $0.3668(16)$ & $0.119(2)$ & $0.185(2)$ & $0.033^{*}$ \\
\hline & & & &
\end{tabular}

๖ Open Access. ( 2018 Jia-Le Li et al., published by De Gruyter. (c) BY-NC-ND This work is licensed under the Creative Commons AttributionNonCommercial-NoDerivatives 4.0 License. 
container and heated at $453 \mathrm{~K}$ for $72 \mathrm{~h}$. After cooling to room temperature within $12 \mathrm{~h}$, purple crystals were obtained in $62 \%$ yield. Analysis calculated (\%): C, 46.43; H, 3.51; N, 32.49\%; Found: C, 46.62; H, 3.36; N, 32.28\%.

\section{Experimental details}

$\mathrm{H}$ atoms bonded to $\mathrm{C}$ atoms were placed geometrically and treated as riding, with $\mathrm{C}-\mathrm{H}$ distances $0.93 \AA$ and $U_{\text {iso }}(\mathrm{H})=1.2 U_{\text {eq }}(\mathrm{C})$ for the $\mathrm{CH}$ while $U_{\text {iso }}(\mathrm{H})=1.2 U_{\text {eq }}(\mathrm{N})$ for the $\mathrm{NH}$ group. The water $\mathrm{H}$ atoms were located from difference maps and refined with the $\mathrm{O}-\mathrm{H}$ distances restrained to $0.82 \AA$ and $U_{\text {iso }}(\mathrm{H})=1.2 U_{\text {eq }}(0)$.

\section{Comment}

Design and synthesis of porous MOFs has been attracted continuous and increasing attention over the past few decades [4-6]. Generally, rational design/choice of organic linkers and inorganic moieties are crucial for the assembly of targeted MOFs with desired structures and properties. Among the various organic ligands, the classes of polydentate N-heterocyclic bases (such as imidazoles, triazoles, tetrazoles and pyrazoles) and aromatic polycarboxylic acids have been broadly explored as building blocks and linkers for the construction of coordination networks [7-9]. In this context, we are particularly interested in the design and construction of MOFs from polydentate N-heterocyclic bases with specific functions such as gas adsorption, luminescence and magnetic properties [10-12]. In our previous study, we have synthesized a novel multi-N containing ligand HL and constructed a series of novel MOFs together with carboxylate ligands [13].

In the title compound, the asymmetric unit contains one 1-(1H-imidazol-4-yl)-3-(4H-tetrazol-5-yl)benzene organic molecule, a half of cobalt(II) cation and one coordinated water molecule (figure). The Co1 is located on an inversion center and has octahedral coordination geometry defined by four nitrogen atoms from four $L^{-}$ligands and two oxygen donors from two water molecules. Each organic deprotonated $L^{-}$as a linear ditopic linker connects two Co(II) atom, leading to an infinite chain with Co ... Co distances of $11.325 \AA$.

Acknowledgements: We gratefully acknowledge support by the Natural Science Foundation of Anhui Provincial Education Commission (No. KH2016B123).

\section{References}

1. BRUKER. SAINT, APEX2 and SADABS. Bruker AXS Inc., Madison, Wisconsin, USA (2009).

2. Sheldrick, G. M.: SHELXT - Integrated space-group and crystal-structure determination. Acta Crystallogr. A71 (2015) 3-8.

3. Sheldrick, G. M.: Crystal structure refinement with SHELXL. Acta Crystallogr. C71 (2015) 3-8.

4. Hu, F.; Di, Z.; Lin, P.; Huang, P.; Wu, M.; Jiang, F.; Hong, M.: An anionic uranium-based metal-organic framework with ultralarge nanocages for selective dye adsorption. Cryst. Growth Des. 18 (2018) 576-580.

5. Furukawa, H.; Ko, N.; Go, Y. B.; Aratani, N.; Choi, S. B.; Choi, E.; Yazaydin, A.; Snurr, R. Q.; O’Keeffe, M.; Kim, J.; Yaghi, O. M.: Ultrahigh porosity in metal-organic frameworks. Science 329 (2010) 424-428.

6. Song, F.; Wang, C.; Falkowski, J. M.; Ma, L.; Lin, W.: Isoreticular chiral metal-organic frameworks for asymmetric alkene epoxidation: tuning catalytic activity by controlling framework catenation and varying open channel sizes. J. Am. Chem. Soc. 132 (2010) 15390-15398.

7. Zhu, M. A.; Guo, X. Z.; Chen, S. S.: Synthesis, crystal structure and luminescent property of a $\mathrm{Zn}$ (II) complex based on 4-imidazole-carboxylate ligand. Chin. J. Struct. Chem. 36 (2017) 1348-1354.

8. Zhu, M. A.; Guo, X. Z.; Xiao, L.; Chen, S. S.: A new Cd(II) coordination compound based on 4-(1,2,4-triazol-4-yl)phenylacetic acid: synthesis, structure and photoluminescence property. Chin. J. Struct. Chem. 37 (2018) 437-444.

9. Zhang, Z. Y.; Xiao, L.; Chen, S. S.; Qiao, R.; Yang, S.: A novel $\mathrm{Zn}$ (II) complex with 4-connected umc topology: synthesis, crystal structure and luminescent property. Chin. J. Struct. Chem. 36 (2017) 819-824.

10. Chen, S. S.; Liu, Q.; Zhao, Y.; Qiao, R.; Sheng, L. Q.; Liu, Z. D.; Yang, S.; Song, C. F.: New metal-organic frameworks constructed from the 4-imidazole-carboxylate ligand: structural diversities, luminescence, and gas adsorption properties. Cryst. Growth Des. 14 (2014) 3727-3741.

11. Chen, S. S.; Wang, P.; Takamizawa, S.; Okamura, T. A.; Chen, M.; Sun, W. Y.: Zinc" and cadmium" metal-organic frameworks with 4-imidazole containing tripodal ligand: sorption and anion exchange properties. Dalton Trans. 43 (2014) 6012-6020.

12. Xiao, L.; Li, W. D.; Fang, X.; Jiang, L. Y.; Chen, S. S.: Two threedimensional supramolecular polymer built from mixed $\mathrm{N}$-donor and carboxylate ligands. Chin. J. Struct. Chem. 35 (2016) 781-788.

13. Chen, S. S.; Qiao, R.; Sheng, L. Q.; Zhao, Y.; Yang, S.; Chen, M. M.; Liu, Z. D.; Wang, D. H.: Cadmium" and zinc" complexes with rigid 1-(1H-imidazol-4-yl)-3-(4H-tetrazol-5-yl) benzene and varied carboxylate ligands. CrystEngComm 15 (2013) 5713-5725. 